\title{
Ionic Cable
}

Can Hui Yang, Baohong Chen, Jing Jing Lu, Jian Hai Yang, Jinxiong Zhou, Yong Mei Chen*, and Zhigang Suo*

Dr. C. H. Yang, ${ }^{[+]}$, Dr. B. Chen, ${ }^{[+]}$, Prof. J. Zhou, 1 State Key Laboratory for Strength and Vibration of Mechanical Structures, International Center for Applied Mechanics, School of Aerospace, Xi'an Jiaotong University, Xi'an, 710049, China.

M.S. J. J. Lu, Dr. J. H. Yang, Prof. Y. M. Chen, 1 Department of Chemistry, School of Science, MOE Key Laboratory for Non-Equilibrium Synthesis and Modulation of Condensed Matter and Department of Chemistry, Xi'an Jiaotong University, Xi' an, 710049, China.

E-mail: chenym@,mail.xjtu.edu.cn

Prof. Z. Suo

1 Kavli Institute for Bionano Science and Technology, Harvard University, Cambridge, MA 02138, USA.

2 School of Engineering and Applied Science, Harvard University, Cambridge, MA 02138, USA.

E-mail: $\underline{\text { suo@seas.harvard.edu }}$

${ }^{[+]}$The first two authors contributed equally to this work.

Keywords: ionic cable, stretchable, transparent, ionic conductors

\begin{abstract}
This paper demonstrates a new type of interconnects to fulfill the primary function of axons: transmitting electrical signals over long distances and at high speeds. The interconnect, which we call "ionic cable", uses ions to transmit signals, and is built entirely with soft, elastic materials - elastomers and gels. The ionic cable is highly transparent, and remains functioning after being stretched nearly eight times its original length. We describe the design, theory and experiment of the ionic cable. We show that the diffusivity of signals in the ionic cable is about 16 orders of magnitude higher than the diffusivity of ions. We demonstrate that the ionic cable transmits signals up to $100 \mathrm{MHz}$ over $10 \mathrm{~cm}$, and transmits music signals over meters. The ionic cable transmits enough power to turn on light-emitting diodes. Our theory shows that the ionic cables scale well, suggesting tremendous opportunities to create miniaturized ionic circuit.
\end{abstract}




\section{Introduction}

In a robot and in an animal, the functions of sensing, deciding and acting usually locate in different places. Whereas the robot coordinates these functions using metallic wires, the animal does so using axons. The metallic wires are hard, but the axons are soft. This difference has inspired the development of stretchable electronics, devices that use patterned electronic conductors to achieve stretchability [1-4]. Recent work demonstrates examples of promising applications. Stretchable conductors replace dangling wires to connect a microprocessor to the fingers of a humanoid, or to the knees of an exoskeleton $[5,6]$. Wearable sensors are placed in contact with the skin of the user to collect healthcare data [7]. Electroceutical devices deliver electrical stimuli to neurons in rats and restore locomotion after paralyzing spinal cord injury [8]. A second difference between metallic wires and axons is also significant: metallic wires transmit signals using electrons, but axons transmit signals using ions. This difference has inspired the recent development of stretchable ionics, devices that use ionic conductors (such as hydrogels and ionogels) to achieve stretchability [9-12].

The stretchable ionic devices demonstrated so far have been actuators and sensors. Here we describe the design, theory and experiment of a family of ionic interconnect, which we call "ionic cable". The ionic cable mimics the function of an axon, not its anatomy. The axon transmits an action potential from head to toe at a speed over $100 \mathrm{~m} / \mathrm{s}$, while maintaining the amplitude of the action potential [13]. The action potential does so by continuously drawing energy through depolarizing the membrane of the axon. By contrast, the signal in our ionic cable will decay. We demonstrate, however, that the decay can be negligible. The ionic cable is analogous to a segment of an axon insulated by myelin sheath [13]. We show that the diffusivity of signals in the ionic cable is about 16 orders of magnitude higher than the diffusivity of ions. We demonstrate that the ionic cable transmits signals up to $100 \mathrm{MHz}$ over $10 \mathrm{~cm}$, and transmits music signals over meters. The ionic cable is highly transparent and stretchable; it remains functioning after being stretched nearly eight times its original length. 
Furthermore, the ionic cable transmits enough power to turn on light-emitting diodes. Our theory shows that the ionic cables scale well, suggesting opportunities to create miniaturized ionic circuits.

The stretchable ionic conductors integrate the two functions-stretchability and conductivity —at the molecular scale. A gel consists of a polymer network and a solvent (e.g., water or ionic liquids). The polymer network makes the gel a soft elastic solid, and the solvent makes the gel a fast ionic conductor. Gels can be as soft as tissues, and as tough as elastomers [14-16]. Although most hydrogels dry out in open air, hydrogels containing humectants retain water in environment of low humidity [17], and ionogels are non-volatile even in vacuum [10].

By contrast, stretchable electronic conductors are commonly hybrids of very dissimilar materials: elastomeric substrates to allow stretch, and electrical conductors to transmit signal. Commonly used electronic conductors include carbon in various forms (powders, fibers and sheets) [18-21], solid metals containing microcracks or patterned in serpentine shapes [22-24], and liquid metals [25]. These stretchable conductors have led to the demonstration of exciting applications, but their drawbacks have been noted: the stretchability is often limited [26], the repeated stretching and releasing may degrade the components that are not intrinsically stretchable [27], and liquid metals are hard to seal. Besides, these hybrids struggle to meet additional requirements in specific applications, such as biocompatibility in bio-mimetic sensors, and transparency in tunable optics [3, 28, 29]. On the other hand, many electronic conductors have high conductivity and can sustain direct current. These considerations suggest that stretchable ionic conductors and stretchable electronic conductors will find different applications that take advantage of their distinct attributes. The object of this paper is to demonstrate that ionic cables can transmit signals over long distances at high speeds.

\section{Structure and Scaling of Ionic Cable}


The basic design of an ionic cable involves two parallel wires of ionic conductors, insulated from each other by a sheet of dielectric (Fig. 1). One end of the ionic cable serves as the input port, connecting through two electrodes to a signal of time-dependent voltage. The other end of the ionic cable serves as the output port, connecting through two electrodes to a load of impedance $Z$. The interface between an ionic conductor and an electrode forms an electrical double layer (EDL). For an ideal polarized electrode, so long as the applied voltage is within a range, e.g., between $-1 \mathrm{~V}$ and $+1 \mathrm{~V}$, electrons and ions do not cross the interface, no electrochemical reaction occurs, and the EDL behaves like a capacitor [30]. To function without electrochemical reaction, the ionic cable is restricted to transmitting alternating current. Furthermore, the resistivity of ionic conductors is typically several orders of magnitude higher than electronic conductors.

We examine the consequences of these fundamental limits by developing a theory of ionic cable. We model the ionic cable as a special case of transmission line [31]. Let $x$ be the coordinate along the cable, and $t$ be time. The length of the cable is much larger than the spacing between the two wires. Each small segment of the cable behaves like a capacitor, which charges and discharges as the current flows in the wires. At a given time and a given segment of the cable, the charges on the two wires are of the same magnitude and the opposite signs. Let $+q(x, t)$ be the charge per unit length of one wire, $-q(x, t)$ be the charge per unit length of the other wire, and $v(x, t)$ be the voltage between the two wires. The magnitude of the charge relates to the voltage as $\mathrm{q}=\mathrm{Cv}$, where $c$ is the capacitance per unit length of the cable. The electric currents propagate along the two wires are of the same magnitude, but are in the opposite directions. Let $i(x, t)$ be the current along one of the wires. Ohm's law requires that $\partial \mathrm{v} / \partial \mathrm{x}=-\mathrm{ri}$, where $r$ is the sum of the resistances per unit length of the two wires. The conservation of electric charge requires that $\partial \mathrm{i} / \partial \mathrm{x}=-\partial \mathrm{q} / \partial \mathrm{t}$. 
The theory shows that signal in the ionic cable runs fast, despite the high resistivity of ionic conductor. A combination of the above equations shows that the voltage $v(x, t)$ obeys the diffusion equation, $\partial \mathrm{v} / \partial \mathrm{t}=\mathrm{D} \partial^{2} \mathrm{v} / \partial \mathrm{x}^{2}$, with the diffusivity of signal being $\mathrm{D}=1 /(\mathrm{rc}) \approx(\mathrm{bd}) /(\rho \varepsilon)$. Here $b$ is the thickness and $\rho$ the resistivity of the ionic conductor, whereas $d$ is the thickness and $\varepsilon$ the permittivity of the dielectric. Using the orders of magnitude representative of our experiments, $b=d=1 \mathrm{~mm}, \rho=10^{-2} \Omega \mathrm{m}$ and $\varepsilon=10^{-11} \mathrm{~F} / \mathrm{m}$, we estimate the diffusivity of signal, $D=10^{7} \mathrm{~m}^{2} / \mathrm{s}$. We note that the diffusivity of signal is many orders of magnitude higher than the diffusivity of ions in water, $D_{i o n}=10^{-9} \mathrm{~m}^{2} / \mathrm{s}[32$ ]. The enormous diffusivity of signal results from a feature in the design: the ionic cable consists of a conductor and a dielectric. Despite the high resistivity of ionic conductor, the permittivity of the dielectric is low, giving a small time scale, $\rho \varepsilon \sim 10^{-13} \mathrm{~s}$. This time scale, together with the thicknesses of the conductor and dielectric, gives the large diffusivity of signal.

It is the high diffusivity of signal that enables the ionic cable to transmit a signal over long distance and at high frequency. A dimensional analysis of our theory shows that the signal decays negligibly if $1^{2} \omega / \mathrm{D}<<1$, where $\omega$ is the frequency of the signal, and $l$ is the length of the cable. For example, for $l=1 \mathrm{~m}, \omega=10^{6} \mathrm{~Hz}$ and $D=10^{7} \mathrm{~m}^{2} / \mathrm{s}$, the dimensionless number is $l^{2} \omega / \mathrm{D}=0.1$. The ionic cable can, of course, transmit signals of lower frequencies over longer distances.

The theory also identifies another dimensionless number, $|\mathrm{Z}| / \mathrm{rl}$, the ratio of the impedance of the load to the resistance of the cable. When the impedance of the load is too small, the voltage drops across the load is small, and output power is small. When the impedance of the load is too high, the current through the load is small, and output power is 
also small. Given an input signal, the output power maximizes when output impedance of the source equals input impedance of the load.

We ascertain these theoretical findings using an experimental setup (Fig. 1b). For

sinusoidal signals, write $\mathrm{v}(\mathrm{x}, \mathrm{t})=\operatorname{Re}\left[\mathrm{V}(\mathrm{x}) \mathrm{e}^{\mathrm{j} \omega \mathrm{t}}\right\rfloor$ and $\mathrm{i}(\mathrm{x}, \mathrm{t})=\operatorname{Re}\left[\mathrm{I}(\mathrm{x}) \mathrm{e}^{\mathrm{j} \omega \mathrm{t}}\right\rfloor$, where $\mathrm{V}(\mathrm{x})$ is the phasor of the voltage, and $\mathrm{I}(\mathrm{x})$ is the phasor of the current. The input port connects to a signal of the phasor $\mathrm{V}(0)$. The output port connects to a load of impedance $Z$, so that $\mathrm{V}(\mathrm{l})=\mathrm{Z}(\mathrm{l})$. We plot solutions when the load is a resistor, an inductor, or a capacitor (Fig. S1-S5). We synthesize a salt-containing hydrogel as ionic conductor, use a commercial elastomer as dielectric (VHB 4905), and use copper as electrodes. We connect the input port to a signal generator (33500B Series, Agilent), connect the output port to a load (a resistor or an inductor), and measure the output voltage and current, $V(l)$ and $I(l)$. The experimental data agree well with theoretical predictions (Fig. 1c, 1d, 1e). Here a low concentration of LiCl $(0.01 \mathrm{~mol} / \mathrm{L})$ is used to obtain an ionic cable with large resistance, so that an obvious decay can be captured within the test frequency (Fig. 1c). However, a high concentration of $\mathrm{LiCl}$ $(8.0 \mathrm{~mol} / \mathrm{L})$ is applied to get a more conductive ionic cable (Fig. 1d, 1e). The ionic cable readily transmits signals up to $100 \mathrm{MHz}$ over $10 \mathrm{~cm}$.

\section{Ionic Music and Ionic Power}

We demonstrate that the ionic cable can transmit music signal (Fig. 2). We cut a commercial electrical cable (Salar, SOMIC Co. Ltd) into two segments, and connect them using an ionic cable. We insert the input port of the electrical cable to a cell phone, and connect the output port to a loudspeaker and an oscilloscope. When the cell phone generates music signal, the ionic cable transmits the signal, the loudspeaker plays the music, and the oscilloscope displays the waveform of the signal (Fig. 2b). The ionic cable is highly transparent and stretchable; it still works well even after being stretched nearly eight times of 
its original length (Fig. 2c, Supplementary Movie 1). Listening to the music, we do not discern any change in the quality of sound as the ionic cable is stretched. Note that stretchability of ionic cable is limited by that of VHB. Altering the thickness or the composition of polyacrylamide hydrogel does not change the stretchability of ionic cable in our experiments. The transparency of ionic cable is also limited by that of VHB due to the exceptionally high transparency and small thickness of polyacrylamide hydrogel within this work. VHB, however, shows high transparency over the range of visible spectrum (Fig. S6). Specially, the ionic cable exhibits a transmittance of $90 \%$ at $550 \mathrm{~nm}$, given the transmittance of polyacrylamide hydrogel $99.99 \%$ and VHB $90.19 \%$ respectively.

The ionic cable readily transmits electrical signals over long distances. We attach an ionic cable of the length $\sim 45.0 \mathrm{~cm}$ to the arm of a person, and the ionic cable transmits music from a mobile phone to the ear (Fig. 2d). The load inside the earphone has inductance $\sim 50$ $\mu \mathrm{H}$. Both experimental results and theoretical analysis indicate that the ionic cable can transmit electrical signals to an inductor (Fig. 1e, S4). A plateau exists over a range of frequency. The signal barely attenuates within the plateau, but decays when the frequency is too small or too large. In spite of the decay (about $95 \%$ for $50 \mu \mathrm{H}$ within the range of audible sound), the music can still be clearly heard from the earphone when the volume of the cell phone is tuned to the maximum.

We next demonstrate that the ionic cable can transmit enough power to turn on lightemitting diodes (Fig. 3). The diodes have a familiar rectifying, nonlinear voltage-current curve (Fig. S7). We connect the input port of the ionic cable to a power source with alternating voltage with peak value of $5.0 \mathrm{~V}$, and connect the output port to the two diodes with antiparallel polarity. This design allows the source of alternating current to power the diodes. When the power source is on, the two diodes light up periodically, in turns (Fig. 3c, Supplementary Movie 2). This demonstration confirms our theoretical prediction and experimental measurement of power-transmitting ionic cables (Fig. 1d, S3b). Within the time 
scale of our experiments, we keep the power source on for more than 24 hours, and the lightemitting diodes keep lighting on periodically without attenuation of brightness. The longevity of ionic cable is sufficient in our experiments.

\section{Concluding Remarks}

Our experiments have demonstrated ionic cables of thickness $\sim 1 \mathrm{~mm}$, and of lengths between $0.1 \mathrm{~m}$ and $10 \mathrm{~m}$. Our theory shows that ionic cables scale well. Recall that $\mathrm{D} \approx(\mathrm{bd}) /(\rho \varepsilon)$, and write the dimensionless number $\mathrm{l}^{2} \omega / \mathrm{D}$ as $\omega \rho \varepsilon \mathrm{l}^{2} /(\mathrm{bd})$. Thus, for a fixed

ratio of impedance $|\mathrm{Z}| / \mathrm{rl}$, ionic cables behave the same if the length and the thicknesses of ionic conductors and dielectrics shrink proportionally. This scaling, along with recent advances in printing gels, suggests tremendous opportunities to create miniaturized ionic circuits.

Life uses mostly ionic circuits, but engineering machines contain mostly electronic circuits. There have been long and practical interests in creating interfaces between life and machines through hybrid ionic-electronic circuits (i.e., ionotronics). Examples include the historical discovery of electricity in frogs, the recording of neural signals (in individual neurons, brains, hearts, etc.), and the development of electroceutical devices. This paper focuses on the fundamental physics of ionic cables, but also demonstrates ionic cables in several simple hybrid ionic-electronic circuits. The ionic cables combine high stretchability and transparency, as well as high diffusivity of signal. It is hoped that this unusual combination of properties will lead to the creation of devices of new functions.

\section{Experimental Section}

Synthesis of polyacrylamide hydrogel containing LiCl: Acrylamide powders and $\mathrm{LiCl}$ grains were dissolved in deionized water, in which the amount of acrylamide was $14.0 \mathrm{wt} \%$ relative to deionized water. The amount of $\mathrm{LiCl}$ was adjusted according to the required 
conductivity of the hydrogel. The crosslinking agent (N,N'-methylenebisacrylamide, MBAA), thermo-initiator (ammonium persulphate, APS) and accelerator (N,N,N',N'tetramethylethylenediamine, TEMED), of molar ratio $0.028 \mathrm{~mol} \%, 0.031 \mathrm{~mol} \%$ and 0.152 mol\%, respectively, relative to acrylamide monomer, were subsequently added into the solution. The mixture became a homogeneous and transparent solution at room temperature. The solution was transferred into a glass mould, which was separated by a silicon spacer. The mould was then put in an oven at $50{ }^{\circ} \mathrm{C}$ for 3 hours to obtain conductive polyacrylamide hydrogel.

Preparation of ionic cable: The synthesized polyacrylamide hydrogels were cut into prescribed length and a sheet of VHB is also cut. Two lines of polyacrylamide hydrogels were attached on the two surfaces of VHB separately and aligning to each other. Four copper pieces (thickness $0.1 \mathrm{~mm}$ ) were cut and attached to the four terminals of the ionic cable.

Measurement of the capacitance of ionic cable: A sheet of dielectric elastomer (VHB 4905), thickness $0.5 \mathrm{~mm}$ and width $1.0 \mathrm{~cm}$, was covered by two copper sheets on two sides, and a capacitance meter was connected to the two copper sheets to measure the capacitance. For a length of $2.0 \mathrm{~cm}$, the measured capacitance was about $17.0 \mathrm{pF}$, which gives the capacitance per unit length of the ionic cable of about $850.0 \mathrm{pF}$. The capacitance can also be estimated theoretically, $c=\varepsilon S / d$, where the permittivity is $\varepsilon=4.2 \times 10^{-11} \mathrm{~F} / \mathrm{m} \mathrm{[33]}$, the area of the capacitor is $S=2.0 \times 10^{-4} \mathrm{~m}^{2}$, and the thickness of the dielectric is $d=5.0 \times 10^{-4} \mathrm{~m}$. These values give a theoretical value of the capacitance of $16.8 \mathrm{pF}$, which corresponds to a capacitance of $840.0 \mathrm{pF}$ per unit length. The measured data was consistent with the theoretical estimation.

Measurement of resistance of ionic cable: The electrical resistance of an ionic conductor was measured using the four-point method. Two external copper electrodes connected the ends of the ionic conductor to a power source. Two inner copper electrodes 
connected two points in the central part of the ionic conductor to a voltmeter to measure the voltage. A galvanometer was in series with the ionic conductor to measure the current. The ratio of the measured voltage to the measured current determined the electrical resistance of the ionic conductor between the two inner probes. The resistance scales as $R=\rho l / A$, where $\rho$ is the resistivity, $l$ the length and $A$ the cross-sectional area. The resistivity depends on the concentration of LiCl. In particular, $\rho=1.6 \times 10^{-2} \Omega \mathrm{m}$ when the concentration of $\mathrm{LiCl}$ was $8.0 \mathrm{~mol} / \mathrm{L}$, and $\rho=2.0 \Omega \mathrm{m}$ when the concentration of $\mathrm{LiCl}$ was $0.01 \mathrm{~mol} / \mathrm{L}$.

\section{Theory of Ionic Cable}

We model ionic cables as a special case of transmission lines. An ionic cable consists of two parallel wires of an ionic conductor, insulated from each other by a dielectric (Fig. 1a). The electric potential and the current vary from point to point along each wire, and vary in time. The length of the cable is much larger than the spacing between the two wires. Each small segment of the cable behaves like a capacitor, which charges and discharges as the current flows in the wires. At a given time and a given segment of the cable, the charges on the two wires are of the same magnitude and the opposite signs. Consequently, the electric currents propagate along the two wires are of the same magnitude, but are in the opposite directions.

Let $x$ be the coordinate along the cable, and $t$ be the time. Let $v(x, t)$ be the voltage between the two wires, and $i(x, t)$ be the current along one of the wires. Ohm's law requires that

$$
\frac{\partial \mathrm{v}}{\partial \mathrm{x}}=-\mathrm{ri}
$$

where $r$ is the sum of the resistances per unit length of the two wires. The charge per unit length of one wire is $+q(x, t)$, and the charge per unit length of the other wire is $-q(x, t)$. The 
conservation of electric charge requires that $\partial \mathrm{i} / \partial \mathrm{x}=-\partial \mathrm{q} / \partial \mathrm{t}$. The magnitude of the charge relates to the voltage as $\mathrm{q}=\mathrm{Cv}$, where $c$ is the capacitance per unit length of the cable. Thus,

$$
\frac{\partial \mathrm{i}}{\partial \mathrm{x}}=-\mathrm{C} \frac{\partial \mathrm{v}}{\partial \mathrm{t}}
$$

Eliminating the current from the above two equations, we find that the voltage $v(x, t)$ obeys the diffusion equation, with the coefficient of diffusion being $1 /$ (rc). To determine $v(x, t)$, we need to prescribe the boundary conditions on the two ports of the cable, as well as the initial condition $v(x, 0)$ along the cable.

When the voltage and the current are sinusoidal in time, we write

$$
\begin{aligned}
& v(x, t)=\operatorname{Re}\left[V(x) e^{j \omega t}\right\rfloor \\
& i(x, t)=\operatorname{Re}\left[I(x) e^{j \omega t}\right\rfloor
\end{aligned}
$$

where $\omega$ is the frequency, $\mathrm{V}(\mathrm{x})$ the phasor of the voltage, and $\mathrm{I}(\mathrm{x})$ the phasor of the current. The phasors are complex-valued, and depend on $x$ but not on $t$. In terms of the phasors, equation (1) and (2) become

$$
\begin{aligned}
& \frac{d V}{d x}=-r I \\
& \frac{d I}{d x}=-j \omega c V
\end{aligned}
$$

This set of ordinary differential equations for the phasors $V(x)$ and $I(x)$ are coupled, linear, and homogeneous. The general solution is

$$
\begin{aligned}
& V(x)=A e^{x \sqrt{j \omega c r}}+B e^{-x \sqrt{j \omega c r}} \\
& I(x)=\frac{\sqrt{j \omega c r}}{r}\left[-A e^{x \sqrt{j \omega c r}}+B e^{-x \sqrt{j \omega c r}}\right]
\end{aligned}
$$


where $A$ and $B$ are constants of integration. Note an identity of complex numbers: $\sqrt{\mathrm{j}}=(1+\mathrm{j}) / \sqrt{2}$

We prescribe the boundary conditions as follows. At the input port, we connect the two terminals to a source of a sinusoidal signal, with a prescribed phasor $\mathrm{V}(0)$ and frequency $\omega$. At the output port, we connect the two terminals to a load of impedance $Z$, so that $\mathrm{V}(\mathrm{l})=\mathrm{Z}(\mathrm{l})$, where $l$ is the total length of the cable. The boundary conditions determine the two constants of integration:

$$
\begin{gathered}
A=\frac{\left(\frac{Z \sqrt{j \omega C r}}{r}-1\right) e^{-1 \sqrt{j \omega c r}} V(0)}{\left(\frac{Z \sqrt{j \omega C r}}{r}+1\right) e^{1 \sqrt{j \omega c r}}+\left(\frac{Z \sqrt{j \omega C r}}{r}-1\right) e^{-1 \sqrt{j \omega c r}}} \\
B=\frac{\left(\frac{Z \sqrt{j \omega C r}}{r}+1\right) e^{1 \sqrt{j \omega c r}} V(0)}{\left(\frac{Z \sqrt{j \omega c r}}{r}+1\right) e^{1 \sqrt{j \omega c r}}+\left(\frac{Z \sqrt{j \omega c r}}{r}-1\right) e^{-1 \sqrt{j \omega c r}}}
\end{gathered}
$$

When the load is a resistor, namely $Z=R$, note that $\frac{Z \sqrt{\omega c r}}{r}=\left(\frac{R}{r l}\right)(l \sqrt{\omega c r})$. The ratio $\mathrm{V}(\mathrm{l}) / \mathrm{V}(0) \mid$ decreases as the frequency increases or as the impedance of the load decreases (Fig. S1). Also plotted are $|I(l) r l / V(0)|$ as functions of frequency or resistance. $|I(l) r l / V(0)|$ decreases as the resistance increases or as the frequency increases (Fig. S2).

The cable transmits power. At a given time $t$ and at a given cross section $x$ of the cable, one wire carries current forward, and the other wire carries current backward; the magnitude of the two currents are the same, $i(x, t)$. The electric potential between the two wires drops by the voltage $v(x, t)$. Consequently, at time $t$, the cable transmits across the section $x$ an amount of power: 


$$
p(x, t)=i(x, t) v(x, t)
$$

When the signal is sinusoidal in time, the power averaged over time is

$$
\mathrm{P}(\mathrm{x})=\frac{\omega}{2 \pi} \int_{0}^{2 \pi \omega} \mathrm{p}(\mathrm{x}, \mathrm{t}) \mathrm{dt}
$$

We can also express the average power in terms of the phasors:

$$
\mathrm{P}(\mathrm{x})=\frac{1}{2} \operatorname{Re}[\mathrm{I}(\mathrm{x}) \overline{\mathrm{V}}(\mathrm{x})]
$$

Here $\overline{\mathrm{V}}$ means the complex conjugate of $V$. The ratio $\mathrm{P}(\mathrm{l}) / \mathrm{P}(0)$ measures how effective the cable transmits power from the input port to the output port. $\mathrm{P}(\mathrm{l}) / \mathrm{P}(0)$ decreases monotonously as the frequency increases while a maximum of $\mathrm{P}(\mathrm{l}) / \mathrm{P}(0)$ exists over the range of variation of resistance (Fig. S3). When the frequency is too high, the ionic cable fails to transmit power, so that $\mathrm{P}(\mathrm{l}) / \mathrm{P}(0)$ is approaching to 0 .

Similarly, consider that the load is an inductor, $Z=j \omega L$. Note that $\frac{Z \sqrt{\omega c r}}{r}=\left(j \frac{L}{c r^{2} l^{3}}\right)(l \sqrt{\omega c r})^{3}$. For a given inductance, a maximum value of $|V(l) / V(0)|$ or $|I(l) r l / V(0)|$ is obtained at an intermediate frequency, and the maximum value drifts as the inductance varies (Fig. S4). When the load is a capacitor, $Z=1 / j \omega C$. Note that $\frac{Z \sqrt{\omega c r}}{r}=\left(\frac{1}{j} \frac{1}{C / c l}\right)\left(\frac{1}{l \sqrt{\omega c r}}\right) \cdot|\mathrm{V}(\mathrm{l}) / \mathrm{V}(0)|$ decreases monotonously as the frequency increases while the maximum value of $|I(l) r l / V(0)|$ is obtained at an intermediate frequency (Fig. S5).

\section{A List of Symbols}

The symbols are listed below according to the sequence they occur in the text. 
$q$ - charge per unit length of the wire;

$c$ - capacitance per unit length of the cable;

$r$ - sum of the resistances per unit length of two wires;

$b$ - thickness of ionic conductor;

$d$ - thickness of dielectric;

$\omega$ - frequency of signal;

$V$ - phasor of voltage; $v$ - voltage between two wires;

$i$ - current along the wire;

$D$ - diffusivity of signal in the cable;

$\rho$ - resistivity of ionic conductor;

$\varepsilon$ - permittivity of dielectric;

$l$ - length of the cable;

$I$ - phasor of current.

\section{Acknowledgements}

This research was supported by the National Natural Science Foundation of China (Grant 51173144, 51073127,11372239, 11472210 and 11321062), International Science \& Technology Cooperation Program supported by Ministry of Science and Technology of China and Shaanxi Province (2013KW14-02), the Research Fund for the Doctoral Program of Higher Education of China, the Scientific Research Foundation for the Returned Overseas Chinese Scholars, State Education Ministry, the Fundamental Research Funds for the Central Universities, the Program for Key Science and Technology Innovative Team of Shaanxi Province (No. 2013KCT-05). ZS acknowledges the support of NSF MRSEC (DMR-0820484) and NSF CMMI-1404653, as well as a visiting appointment at the International Center for Applied Mechanics. 


\section{References}

[1] S. Wagner, S. P. Lacour, J. Jones, P. H. I. Hsu, J. C. Sturm, T. Li, Z. G. Suo, Phys. E 2004, 25, 326 .

[2] M. Kaltenbrunner, T. Sekitani, J. Reeder, T. Yokota, K. Kuribara, T. Tokuhara, M.

Drack, R. Schwodiauer, I. Graz, S. Bauer-Gogonea, S. Bauer, T. Someya, Nature 2013, 499, 458.

[3] M. Ramuz, B. C. K. Tee, J. B. H. Tok, Z. Bao, Adv. Mater. 2012, 24, 3223.

[4] M. L. Hammock, A. Chortos, B. C.-K. Tee, J. B.-H. Tok, Z. Bao, Adv. Mater. 2013, $25,5997$.

[5] H. Alirezaei, A. Nagakubo, Y. Kuniyoshi, presented at Proc. 7th IEEE-RAS Int. Conf. Humanoid Robots, Pittsburgh, PA, 2007.

[6] J. T. B. Overveldea, Y. Menguc, P. Polygerinos, Y. Wang, Z. Wang, R. J. Wood, C. J. Walsh, K. Bertoldi, Extreme Mechanics Letters, http:// dx.doi.org/10.1016/j.eml.2014.11.003.

[7] D. H. Kim, N. Lu, R. Ma, Y. S. Kim, R. H. Kim, S. Wang, J. Wu, S. M. Won, H. Tao, A. Islam, K. J. Yu, T. Kim, R. Chowdhury, M. Ying, L. Xu, M. Li, H. J. Chung, H. Keum, M. McCormick, P. Liu, Y. W. Zhang, F. G. Omenetto, Y. Huang, T. Coleman, J. A. Rogers, Science 2011, 333, 838 .

[8] I. R. Minev, P. Musienko, A. Hirsch, Q. Barraud, N. Wenger, E. M. Moraud, J. Gandar, M. Capogrosso, T. Milekovic, L. Asboth, R. F. Torres, N. Vachicouras, Q. Liu, N. Pavlova, S. Duis, A. Larmagnac, J. Voros, S. Micera, Z. Suo, G. Courtine, S. P. Lacour, Science 2015, 347, 159.

[9] C. Keplinger, J. Y. Sun, C. C. Foo, P. Rothemund, G. M. Whitesides, Z. Suo, Science 2013, 341, 984 .

[10] B. Chen, J. J. Lu, C. H. Yang, J. H. Yang, J. Zhou, Y. M. Chen, Z. Suo, ACS Appl. Mater. Interfaces 2014, 6, 7840. 
[11] Y. Bai, Y. Jiang, B. Chen, C. C. Foo, Y. Zhou, F. Xiang, J. Zhou, H. Wang, Z. Suo, Appl. Phys. Lett. 2014, 104, 062902.

[12] J. Y. Sun, C. Keplinger, G. M. Whitesides, Z. Suo, Adv. Mater. 2014, 26, 7608.

[13] M. F. Bear, B. W. Conners, M. A. Paradiso, Neuroscience, Lippincott Williams \& Wilkins, 2007.

[14] J. P. Gong, Y. Katsuyama, T. Kurokawa, Y. Osada, Adv. Mater. 2003, 15, 1155.

[15] J. Y. Sun, X. Zhao, W. R. K. Illeperuma, O. Chaudhuri, K. H. Oh, D. J. Mooney, J. J. Vlassak, Z. Suo, Nature 2012, 489, 133.

[16] C. H. Yang, M. X. Wang, H. Haider, J. H. Yang, J. Y. Sun, Y. M. Chen, J. Zhou, Z. Suo, ACS Appl. Mater. Interfaces 2013, 5, 10418.

[17] Y. Bai, B. Chen, F. Xiang, J. Zhou, H. Wang, Z. Suo, Appl. Phys. Lett. 2014, 105, 151903.

[18] S. Park, M. Vosguerichian, Z. Bao, Nanoscale 2013, 5, 1727.

[19] T. A. Kim, H. S. Kim, S. S. Lee, M. Park, Carbon 2012, 50, 444.

[20] S. Rosset, H. R. Shea, Appl. Phys. A 2013, 110, 281.

[21] M. F. L. D. Volder, S. H. Tawfick, R. H. Baughman, A. J. Hart, Science 2013, 339, 535.

[22] S. P. Lacour, S. Wagner, Z. Y. Huang, Z. Suo, Appl. Phys. Lett. 2003, 82, 2404.

[23] S. Xu, Y. Zhang, J. Cho, J. Lee, X. Huang, L. Jia, J. A. Fan, Y. Su, J. Su, H. Zhang, H. Cheng, B. Lu, C. Yu, C. Chuang, T. Kim, T. Song, K. Shigeta, S. Kang, C. Dagdeviren, I.

Petrov, P. V. Braun, Y. Huang, U. Paik, J. A. Rogers, Nat. Commun. 2013, 4, 1543.

[24] D. J. Lipomi, Z. Bao, Energy Environ. Sci. 2011, 4, 3314.

[25] S. Zhu, J. H. So, R. Mays, S. Desai, W. R. Barnes, B. Pourdeyhimi, M. D. Dickey, Adv. Funct. Mater. 2013, 23, 2308.

[26] X. Feng, B. D. Yang, Y. Liu, Y. Wang, C. Dagdeviren, Z. Liu, A. Carlson, J. Li, Y. Huang, J. A. Rogers, ACS Nano 2011, 5, 3326. 
[27] D. Y. Khang, J. A. Rogers, H. H. Lee, Adv. Funct. Mater. 2008, $1,1$.

[28] D. H. Kim, J. Viventi, J. J. Amsden, J. Xiao, L. Vigeland, Y. S. Kim, J. A. Blanco, B. Panilaitis, E. S. Frechette, D. Contreras, D. L. Kaplan, F. G. Omenetto, Y. Huang, K. C. Hwang, M. R. Zakin, B. Litt, J. A. Rogers, Nat. Mater. 2010, 9, 511.

[29] S. Shian, R. M. Diebold, D. R. Clarke, Opt. Express 2013, 21, 8669.

[30] A. J. Bard, L. R. Faulkner, Electrochemical methods, John Wiley \& Sons, Inc. 2001.

[31] R. Bansal, Handbook of Engineering Electromagnetics, University of Connecticut Storrs, Connecticut, USA 2004.

[32] Y. H. Li, S. Gregory, Geochim. Cosmochim. Acta. 1974, 38, 703.

[33] B. Chen, Y. Bai, F. Xiang, J. Y. Sun, Y. M. Chen, H. Wang, J. Zhou, Z. Suo, J. Polym. Sci., Part B: Polym. Phys. 2014, 52, 1055. 
a

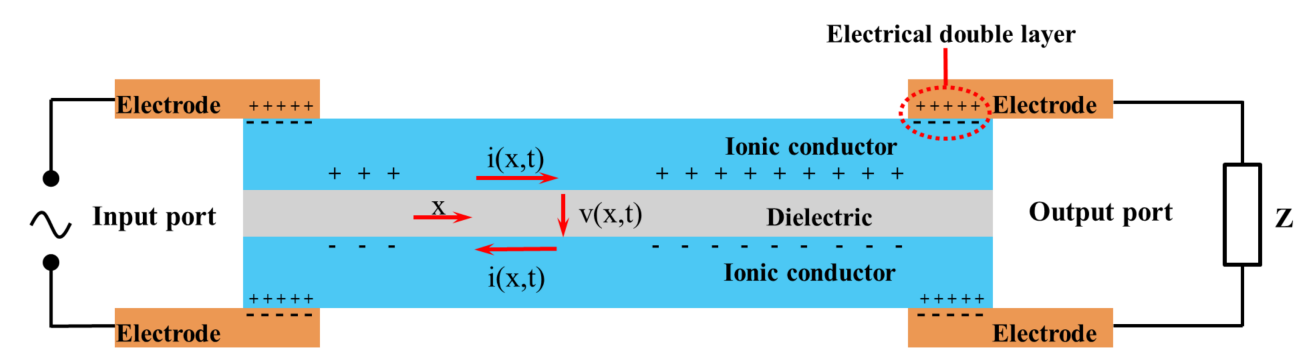

b

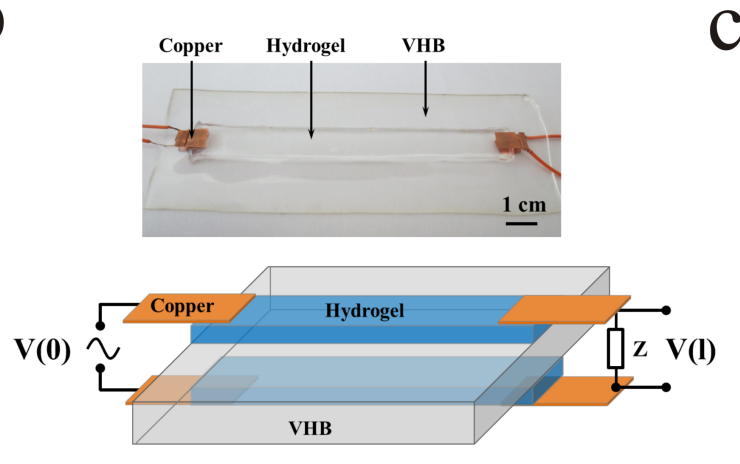

d

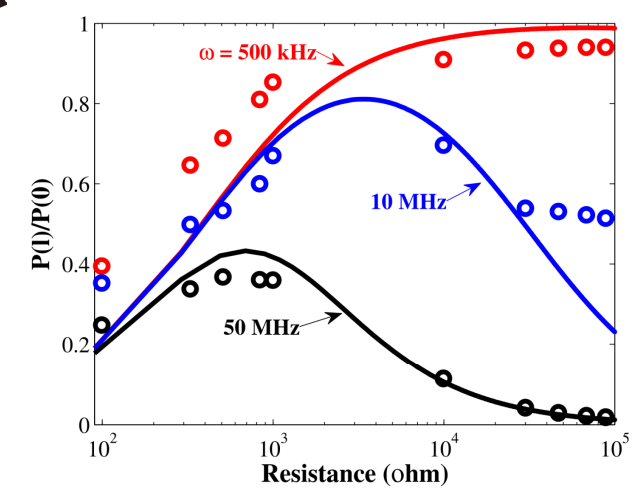

$\mathrm{C}$

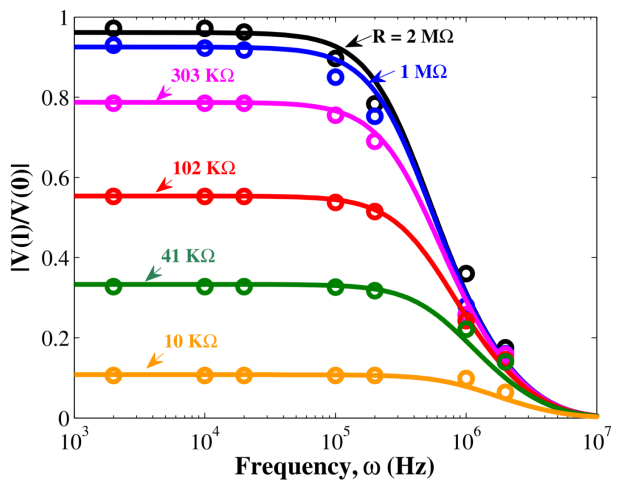

e

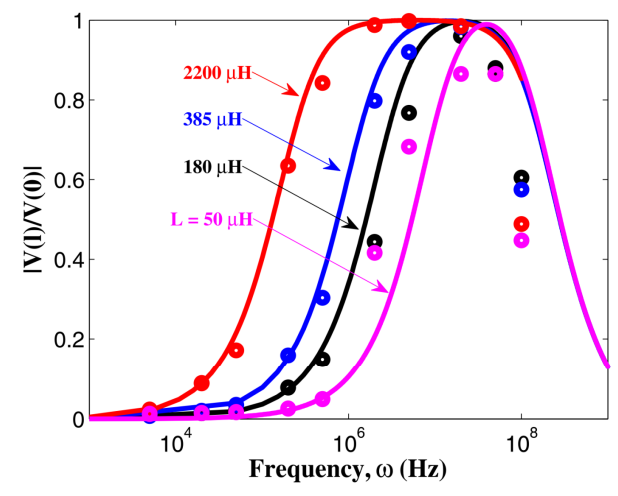

Fig. 1. Structure and electrical characteristics of an ionic cable. (a) A basic design of ionic cable. (b) Experimental setup. Two strips of LiCl-containing polyacrylamide hydrogel (length $10 \mathrm{~cm}$, width $1 \mathrm{~cm}$, thickness $0.5 \mathrm{~mm}$ ) are insulated by a sheet of dielectric elastomer (VHB 4905, thickness $0.5 \mathrm{~mm}$ ). (c) Theoretical results (solid curves) and experimental results (open circles) of $|\mathrm{V}(\mathrm{l}) / \mathrm{V}(0)|$ vary with frequency for several fixed resistors. Here we use a low concentration of $\mathrm{LiCl}(0.01 \mathrm{~mol} / \mathrm{L})$ to obtain an ionic cable with large resistance, so that an obvious decay can be captured within the test frequency. (d) The ratio $\mathrm{P}(\mathrm{l}) / \mathrm{P}(0)$ varies with resistance for several fixed frequencies. (e) The ratio $|V(l) / V(0)|$ varies with frequency for several fixed inductors. For (d) and (e), the thickness of hydrogel strips is $2.0 \mathrm{~mm}$ and the concentration of $\mathrm{LiCl}$ is $8.0 \mathrm{~mol} / \mathrm{L}$. 
a

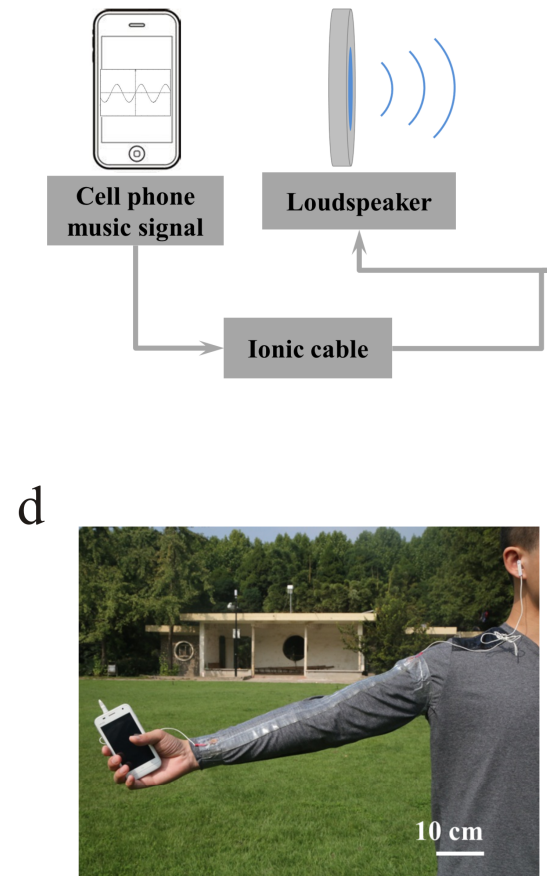

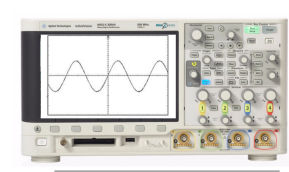

Oscilloscope

b

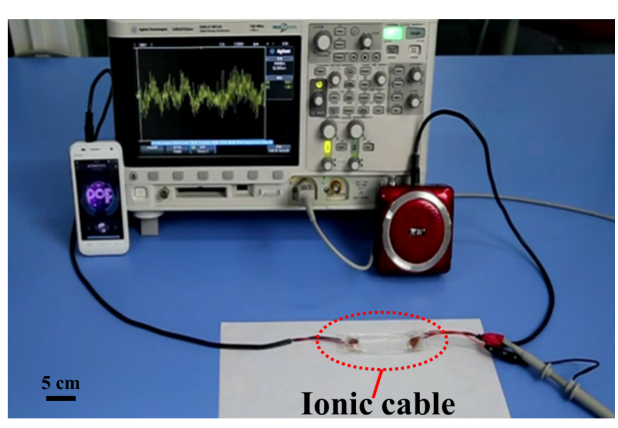

C
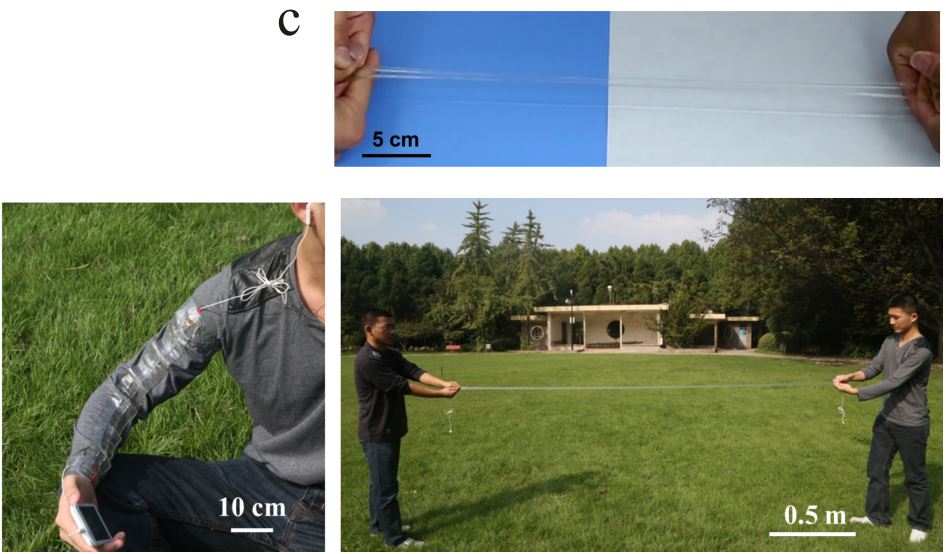

Fig. 2. Stretchable, transparent, ionic cable transmits music signal. (a) Schematic of the experimental setup. The electrical signal from the cell phone is fed through an ionic cable to a loudspeaker and an oscilloscope. (b) Image of the experimental setup. (c) The ionic cable is highly stretchable and transparent and still works even when it is stretched up to nearly eight times its original length. (d) An ionic cable $(\sim 45.0 \mathrm{~cm})$ is attached along the arm of a person, and transmits music signal from a cell phone to the ear. The polyacrylamide hydrogels are 2.0 $\mathrm{mm}$ thick and the concentration of $\mathrm{LiCl}$ is $8.0 \mathrm{~mol} / \mathrm{L}$. 
a

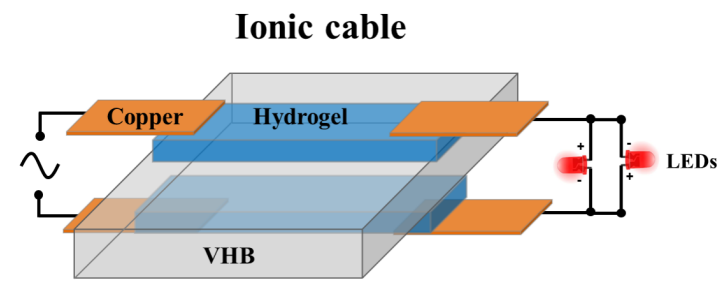

b

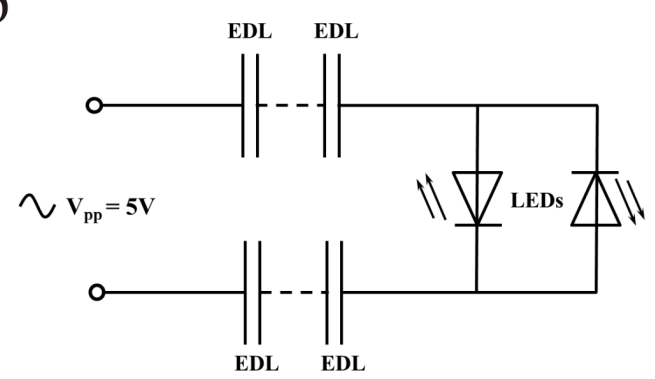

C

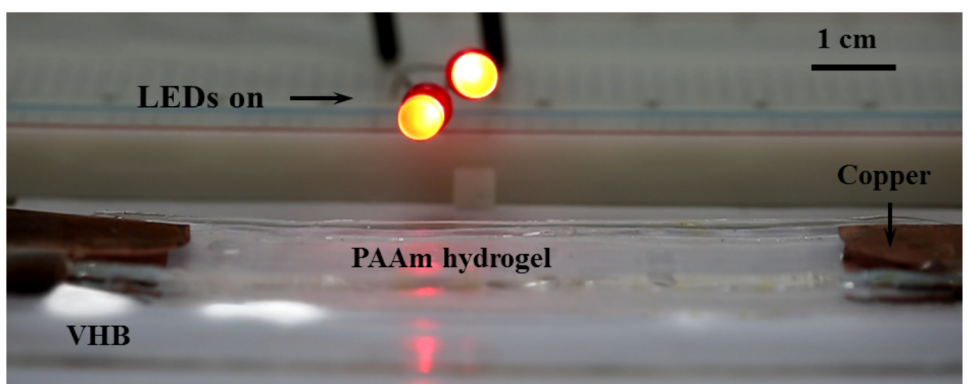

Fig. 3. Ionic cable transmits power to turn on light-emitting diodes. (a) Schematic of the experimental setup. (b) Diagram of the circuit. The dashed lines represent the ionic conductors. (c) Image of the illuminated diodes (TLHR5400). The polyacrylamide hydrogel is $2.0 \mathrm{~mm}$ thick and the concentration of $\mathrm{LiCl}$ is $8.0 \mathrm{~mol} / \mathrm{L}$. 